\title{
Aharonov-Bohm effect in the chiral Luttinger liquid
}

\author{
Michael R. Geller ${ }^{1}$ and Daniel Loss ${ }^{2}$ \\ ${ }^{1}$ Department of Physics, Simon Fraser University, Burnaby B.C. V5A 1S6, Canada \\ ${ }^{2}$ Department of Physics, University of Basel, Klingelbergstrasse 82, 4056 Basel, Switzerland
}

(July 8, 2021)

\begin{abstract}
Edge states of the quantum Hall fluid provide an almost unparalled opportunity to study mesoscopic effects in a highly correlated electron system. In this paper we develop a bosonization formalism for the finite-size edge state, as described by chiral Luttinger liquid theory, and use it to study the Aharonov-Bohm effect. The problem we address may be realized experimentally by measuring the tunneling current between two edge states through a third edge state formed around an antidot in the fractional quantum Hall effect regime. The finite size $L$ of the antidot edge state introduces a temperature scale $T_{0} \equiv \hbar v / \pi k_{B} L$, where $v$ is the edge-state Fermi velocity. A renormalization group analysis reveals the existence of a two-parameter universal scaling function $\tilde{G}(X, Y)$ that describes the Aharonov-Bohm resonances. We also show that the strong renormalization of the tunneling amplitudes that couple the antidot to the incident edge states, together with the nature of the Aharonov-Bohm interference process in a chiral system, prevent the occurrence of perfect resonances as the magnetic field is varied, even at zero temperature. In an experimentally realizable strong-antidot-coupling regime, where the source-to-drain transmission is weak, and at bulk filling factor $g=1 / q$ with $q$ an odd integer, we predict the low-temperature $\left(T \ll T_{0}\right)$ Aharonov-Bohm amplitude to vanish with temperature as $T^{2 q-2}$, in striking contrast to a Fermi liquid $(q=1)$. Near $T_{0}$, there is a pronounced maximum in the amplitude, also in contrast to a Fermi liquid. At high temperatures $\left(T \gg T_{0}\right)$, however, we predict a crossover to a $T^{2 q-1} e^{-q T / T_{0}}$ temperature dependence, which is qualitatively similar to chiral Fermi liquid behavior. Careful measurements in the strong-antidot-coupling regime above $T_{0}$ should be able to distinguish between a Fermi liquid and our predicted nearly Fermi-liquid scaling. In addition, we predict an interesting high-temperature nonlinear response regime, where the voltage satisfies $V>T>T_{0}$, which may also be used to distinguish between chiral Fermi liquid and chiral Luttinger liquid behavior. Finally, we predict new mesoscopic edge-current oscillations, which are similar to the persistent current oscillations in a mesoscopic ring, except that they are not reduced in amplitude by weak disorder. In the fractional quantum Hall regime, these "chiral persistent currents" have a universal non-Fermi-liquid temperature dependence and may be another ideal system to observe a chiral Luttinger liquid.
\end{abstract}

PACS: 73.40.Hm, 71.27.+a, 73.20.Dx, 73.40.Gk

\section{INTRODUCTION}

It is well known that the integer quantum Hall effect [1] and many related transport phenomena that occur in nanometer-scale semiconductor devices [2] can be understood in terms of Fermi liquid theories of magnetic edgestate transport [3], such as the Büttiker-Landauer formalism [4]. However, as was shown by Laughlin [5], the fractional quantum Hall effect (FQHE) occurs because strong electron-electron interactions result in the formation of highly correlated incompressible states at certain Landau level filling factors [1]. In 1990, Wen [6] used the Chern-Simons theory [7] of the bulk FQHE to show that the edge states in the fractional regime should be chiral Luttinger liquids (CLL). As in the nonchiral Luttinger liquid [8,9], electron-electron interactions in the CLL play an essential role and lead to physical properties that can be dramatically different than the integral quantum Hall effect edge state. Wen's important proposal has stimulated a considerable theoretical effort to understand the properties of this non-Fermi-liquid state of matter 1025 .
The first experimental observation of a CLL was made by Milliken, Umbach, and Webb [26]. These authors measured the tunneling current between two filling factor $1 / 3$ edge states in a quantum-point-contact geometry. As the gate voltage was varied, resonance peaks in the conductance, caused by conditions of destructive interference that prevent tunneling between the edges, were observed to have the correct CLL temperature dependence as predicted by Moon and coworkers [13] and by Fendley, Ludwig, and Saleur 17. In addition, Chang, Pfeiffer, and West [27], working with a cleaved-edge sample and measuring the tunneling between a single filling factor $1 / 3$ edge state and doped GaAs, have very recently reported experimental evidence that is also in favor of CLL theory. Thus, it would appear that the CLL is indeed realized in FQHE edge states.

However, two recent experiments 228,29] on the tunneling between FQHE edge states through an additional edge state circling a quantum antidot have reported Fermi liquid behavior. In the quantum-point-contact geometry the tunneling occurs through an unknown and uncontrollable impurity distribution, resulting in a dense 
series of resonance peaks [26]. In contrast, the tunneling in the anitdot system occurs through a larger object, essentially a mesoscopic ring, and the resonances are controlled by the Aharonov-Bohm (AB) effect by varying the magnetic field within a given FQHE plateau. For this reason the antidot geometry is believed to be superior for observing resonant tunneling in a CLL. Franklin et al. [28] measured the $\mathrm{AB}$ conductance oscillations in a device with an antidot $0.94 \mu \mathrm{m}$ in circumference at temperatures down to $30 \mathrm{mK}$. The filling factor in the immediate vicinity of the antidot was measured to be $1 / 3$, whereas in the bulk it was $2 / 3$. In both the integer and fractional regimes the period of the $\mathrm{AB}$ oscillations was observed to correspond to one flux quantum through the area bounded by the antidot edge state. The temperature dependence was analyzed by determining the temperature dependence of the appropriate Fourier coefficient of the transformed resistance data, and a good fit to Fermi liquid scaling was obtained. More recently, Maasilta and Goldman 29], following earlier related work by Goldman and $\mathrm{Su}$ [30], studied resonant tunneling as a function of back-gate voltage down to $12 \mathrm{mK}$ in an antidot $1.9 \mu \mathrm{m}$ in circumference. Within the experimental uncertainty, the resonance lineshape at a given temperature was consistent with both chiral Fermi liquid and CLL theory. The temperature dependence of the width and amplitude of the oscillations, however, was found to be that of a chiral Fermi liquid.

The agreement of these experiments with Fermi liquid theory does not in itself rule out CLL theory because no detailed CLL theory for the antidot geometry has been available. This is one motivation of the present work. The most important conclusion of our study is that the transport properties of the quantum-point-contact system and the antidot system differ in two important ways. The first is that mesoscopic effects are very important in the latter. When the thermal length $L_{\mathrm{T}} \equiv \hbar v / k_{\mathrm{B}} T$ becomes smaller than the circumference $L$ of the antidot edge state, the $\mathrm{AB}$ oscillations become washed out, and, at the same time, acquire a temperature dependence that is similar to a chiral Fermi liquid 24]. Here $v$ is the edge-state Fermi velocity. An experiment performed at a temperature significantly above the point of crossover,

$$
T_{0} \equiv \frac{\hbar v}{\pi k_{\mathrm{B}} L},
$$

is therefore expected to observe nearly Fermi liquid behavior for many mesoscopic quantities. (The precise definition of $T_{0}$ has been chosen to simplify the expressions in Section V.) The second difference is that in contrast with the quantum-point-contact geometry, where it is reasonable to assume that there exist conditions of destructive interference that lead to perfect resonances, the resonances in the antidot geometry, which are controlled by the $\mathrm{AB}$ effect, are never perfect, even at zero temperature. As we shall explain, this means that there is another temperature scale $T_{1}$, set by the bare tunneling rate between the antidot and incident edge states, below which the system is always in the strongly coupled regime.

The other motivation for our work is that the CLL provides an almost unparalled opportunity to study mesoscopic physics in a highly correlated electron system that is both experimentally accessible and theoretically tractable. Thus, our comparison of the AB effect in the Fermi and Luttinger liquids is also a comparison of mesoscopic effects in a noninteracting and interacting system, and we shall show that at temperatures below $T_{0}$ interactions have a dramatic effect on many quantities. Mesoscopic effects in nonchiral Luttinger liquids have been studied considerably 9, 31, 32], and a recent interesting paper by Chamon and coworkers 25] has analyzed mesoscopic effects in a CLL by considering a double pointcontact arrangement that allows one to measure the fractional charge and fractional statistics of FQHE quasiparticles. Although the main focus of their work is different, and the geometry they consider is close to that of a quantum dot instead of an antidot, many of the results of Chamon et al. 25 are similar to ours.

The model of the antidot system we shall adopt for our study is the simplest one possible: We assume two macroscopic filling factor $g=1 / q$ (with $q$ odd) edge states at the edges of the Hall bar symmetrically coupled to a single mesoscopic edge state (with the same $g$ ) circling the antidot. The edges of the Hall fluid are assumed to be sharply confined, and the interaction short-ranged, so that the low lying excitations consist of a single branch of edge-magnetoplasmons with linear dispersion $\omega=v k$. It is not known at present whether or not the deviations of the experimentally studied systems from this idealized model are important.

The original effect of Aharonov and Bohm [33] was proposed as an experiment with electron beams in a vacuum, but it was realized immediately that electrons moving in a multiply connected conductor should also be influenced by a magnetic flux threaded through it. Because it is a sensitive probe of phase coherence, the AB effect has been an important experimental tool to study disordered metals [34]. Before proceeding with our study, it is worthwhile to inquire whether there are any essential differences between the $\mathrm{AB}$ effect in nonchiral and chiral systems. The answer is yes: In nonchiral systems, the AB flux can lead to both constructive and destructive interference, whereas only constructive interference is possible in the chiral system. This difference can be understood in the following way.

Let $\theta_{ \pm} \equiv \oint_{\epsilon} d \mathbf{l} \cdot\left(\mathbf{p}+\frac{e}{c} \mathbf{A}\right)$ be the phase accumulated by an electron with energy $\epsilon$ after one complete clockwise $(+)$ or counterclockwise $(-)$ orbit around the ring shown in Fig. 11; it is given by $\theta_{ \pm}=2 \pi\left(\frac{\epsilon}{\Delta \epsilon} \pm \varphi\right)$ and includes both the dynamical and $\mathrm{AB}$ phases. Here $e$ is the magnitude of the electron charge, $\varphi \equiv \Phi / \Phi_{0}$ is the dimensionless $\mathrm{AB}$ flux threading the ring, $\Phi_{0} \equiv h c / e$ is the flux quantum, and $\Delta \epsilon \equiv 2 \pi v / L$ is the energy level spacing for noninteracting electrons having linear disper- 
sion with velocity $v$ in a ring of circumference $L$. The probability amplitude $\mathcal{A}_{ \pm}$to propagate on the ring from point 1 to point 2 at energy $\epsilon$, allowing only clockwise $(+)$ or counterclockwise $(-)$ motion, is given by

$$
\mathcal{A}_{ \pm}=e^{i \theta / 2}+e^{i 3 \theta / 2}+e^{i 5 \theta / 2}+\cdots=\frac{i / 2}{\sin \pi\left(\frac{\epsilon}{\Delta \epsilon} \pm \varphi\right)} .
$$

The first term in the series, $e^{i \theta / 2}$, is the amplitude to propagate directly from point 1 to point 2 , and the remaining terms account for any number of windings with a given chirality. The total "transmission" probability in the chiral case is therefore

$$
\left|\mathcal{A}_{ \pm}\right|^{2}=\frac{1 / 2}{1-\cos 2 \pi\left(\frac{\epsilon}{\Delta \epsilon} \pm \varphi\right)},
$$

which possesses transmission resonances when $\frac{\epsilon}{\Delta \epsilon} \pm \varphi$ is integral, but never exhibits completely destructive interference. In contrast, the total transmission probability in the nonchiral case,

$$
\left|\mathcal{A}_{+}+\mathcal{A}_{-}\right|^{2}=\frac{\sin ^{2}\left(\pi \frac{\epsilon}{\Delta \epsilon}\right) \cos ^{2}(\pi \varphi)}{\sin ^{2} \pi\left(\frac{\epsilon}{\Delta \epsilon}+\varphi\right) \sin ^{2} \pi\left(\frac{\epsilon}{\Delta \epsilon}-\varphi\right)},
$$

has both poles and zeros. A more precise analysis of this distinction, based on a comparison of the chiral and nonchiral propagators, is provided in Appendix A.

The organization of this paper is as follows. In Section II we study the $\mathrm{AB}$ effect in the chiral Fermi liquid for arbitrary antidot-coupling strength with the BüttikerLandauer formula. In Section III we discuss the general theory of the finite-size CLL, including canonical quantization and bosonization. We also show there how fractionally charged excitations arise naturally in the CLL. Section IV is devoted to a renormalization group analysis of the weak-antidot-coupling regime, where we obtain the same flow equations as previously derived for the quantum-point-contact system. The strong-antidotcoupling regime of the $\mathrm{AB}$ effect in a CLL is studied in detail in Section V. In Section VI we study the response of an edge state to an $\mathrm{AB}$ flux and find mesoscopic edgecurrent oscillations that are similar to persistent current oscillations in a mesoscopic ring, except that they are not degraded by weak disorder. Section VII contains a brief discussion of our results and their relevance to the existing antidot experiments.

\section{AHARONOV-BOHM EFFECT IN THE CHIRAL FERMI LIQUID}

We begin by studying the chiral Fermi liquid case with the Büttiker-Landauer formula, which is valid for noninteracting electrons. In addition to serving as a check of our more general expressions derived below, the BüttikerLandauer formula is valid for arbitrary antidot-coupling amplitude and the resulting conductance exhibits resonances that are not accessible from a perturbation expansion valid for small tunneling. The Büttiker-Landauer analysis also shows that the nature of the $\mathrm{AB}$ resonances in the chiral Fermi liquid and CLL are entirely different: In the weak-antidot-coupling regime of Fig. 2aa, with tunneling amplitudes $\left|\Gamma_{ \pm}\right| \ll 1$, the two-terminal conductance in the Fermi liquid possesses reflection resonances (sharp dips in the conductance) when the electron going around the antidot interferes constructively with itself, whereas, as we shall show below, the chiral Luttinger liquid instead exhibits resonant transmission (sharp peaks in the conductance) at conditions of maximum destructive interference.

Transport through an antidot in the integer quantum Hall effect regime has been studied both theoretically [35 37 and experimentally [38. In the two-terminal Büttiker-Landauer approach, the constriction containing the antidot is regarded as a single phase-coherent scatterer connecting perfect reservoirs serving both as current sources and voltage probes. The current flowing from reservoir 1 to reservoir 2 is

$$
I=-\frac{e}{h} \int d \epsilon T(\epsilon)\left[n_{\mathrm{F}}\left(\epsilon-\mu_{1}\right)-n_{\mathrm{F}}\left(\epsilon-\mu_{2}\right)\right],
$$

where $T(\epsilon)$ is the total probability for transmission from reservoir 1 to $2, n_{\mathrm{F}}(\epsilon) \equiv\left(e^{\beta \epsilon}+1\right)^{-1}$ is the Fermi distribution function, and $\mu_{i}$ is the electrochemical potential of reservoir $i$. The voltage $V \equiv\left(\mu_{2}-\mu_{1}\right) / e$ is defined so that a positive $V$ produces a positive $I$. The two-terminal linear conductance is

$$
G=-\frac{e^{2}}{h} \int d \epsilon \frac{\partial n_{\mathrm{F}}}{\partial \epsilon} T(\mu+\epsilon),
$$

where $\mu \equiv \frac{\mu_{1}+\mu_{2}}{2}$ is the mean electrochemical potential, which reduces to $G=T(\mu) \frac{e^{2}}{h}$ at zero temperature.

The transmission probability $T(\epsilon)$ has been evaluated for a variety of edge state configurations in Ref. [35]. For our purposes it is sufficient to consider only the case where $\Gamma_{ \pm}=i \Gamma$ with $\Gamma$ real and energy independent [39]. In this case the amplitude to tunnel on or off the antidot is $i \Gamma$, whereas, by unitarity, the amplitude to proceed without tunneling is $\sqrt{1-\Gamma^{2}}$. Then for the system shown in Fig. 20a it is simple to show that

$$
T(\epsilon)=1-\frac{\Gamma^{4}}{2\left(1-\Gamma^{2}\right)[1-\cos \theta(\epsilon)]+\Gamma^{4}},
$$

where $\theta=2 \pi\left(\frac{\epsilon}{\Delta \epsilon}+\varphi\right)$ is the phase shift of the electron wave function after a complete clockwise orbit around the antidot at energy $\epsilon$, as defined in Section I, with $\varphi$ now the dimensionless magnetic flux through the area defined by the antidot edge state. (Note that the arrows in Fig. 2 denote the flow of currents; the electrons are therefore circling the antidot in the clockwise direction.)

In the weak-antidot-coupling regime, where $\Gamma \ll 1$, (7) shows that sharp zero-temperature reflection resonances in the two-terminal conductance occur when $\theta=2 \pi n$ 
with $n$ an integer; that is, at conditions of constructive interference. In the strong-antidot-coupling regime, where the tunneling amplitude $\Gamma$ is close to unity, the source-drain transmission probability is small. In this regime it is convenient to define a new small parameter $\bar{\Gamma} \equiv \sqrt{1-\Gamma^{2}}$. Then in the strong-coupling limit $(\bar{\Gamma} \ll 1)$ we have

$$
T(\epsilon)=2 \bar{\Gamma}^{2}[1-\cos \theta(\epsilon)]+\mathcal{O}\left(\bar{\Gamma}^{4}\right) .
$$

Note that reflection resonances still occur when $\theta=2 \pi n$, although they are less sharp than in the weak-antidotcoupling limit. This limit corresponds to the case for which the CLL theory described in Section $V$ has been developed. The zero-temperature $\mathrm{AB}$ resonances in the weak and strong coupling regimes are shown in Fig. 3 .

An analytic expression for the linear and nonlinear response in the strong-antidot-coupling regime can be obtained from (5) and (8). The required integral,

$$
\begin{gathered}
\int_{-\infty}^{\infty} d \epsilon \frac{\cos 2 \pi\left(\frac{\epsilon}{\Delta \epsilon}+\frac{\mu_{1}}{\Delta \epsilon}+\varphi\right)-\cos 2 \pi\left(\frac{\epsilon}{\Delta \epsilon}+\frac{\mu_{2}}{\Delta \epsilon}+\varphi\right)}{e^{\beta \epsilon}+1} \\
=-2 \pi T \frac{\sin \left(\frac{e V}{2 \pi T_{0}}\right) \cos 2 \pi\left(\frac{\mu}{\Delta \epsilon}+\varphi\right)}{\sinh \left(\frac{T}{T_{0}}\right)}
\end{gathered}
$$

follows from a contour integration and residue summation. Here we have used the definitions of $\mu$ and $V$ to write $\mu_{1}=\mu-e V / 2$ and $\mu_{2}=\mu+e V / 2$. In this regime we therefore find for the chiral Fermi liquid

$$
I^{\mathrm{FL}}=I_{0}^{\mathrm{FL}}+I_{\mathrm{AB}}^{\mathrm{FL}} \cos 2 \pi\left(\frac{\mu}{\Delta \epsilon}+\varphi\right),
$$

where

$$
I_{0}^{\mathrm{FL}}=\frac{e^{2} \bar{\Gamma}^{2}}{\pi \hbar} V
$$

is the flux-independent contribution, and

$$
I_{\mathrm{AB}}^{\mathrm{FL}}=-\frac{2 e \bar{\Gamma}^{2}}{\hbar} \frac{T}{\sinh \left(T / T_{0}\right)} \sin \left(\frac{e V}{2 \pi T_{0}}\right)
$$

is the $\mathrm{AB}$ contribution. If the voltage $V$ is applied symmetrically about an antidot energy level then $\mu$ is an integral multiple of $\Delta \epsilon$ and the voltage dependence in the second term of (10) becomes simply $\sin \left(e V / 2 \pi T_{0}\right)=$ $\sin (e V \pi / \Delta \epsilon)$. The voltage dependence is then sinusoidal with a period equal to twice the antidot level spacing $\Delta \epsilon$, because as the voltage is varied the two chemical potentials $\mu_{1}$ and $\mu_{2}$ move in opposite directions at half the rate at which $V$ changes. The linear conductance $G \equiv(d I / d V)_{V=0}$ in this regime is given by

$$
G^{\mathrm{FL}}=G_{0}^{\mathrm{FL}}+G_{\mathrm{AB}}^{\mathrm{FL}} \cos 2 \pi\left(\frac{\mu}{\Delta \epsilon}+\varphi\right),
$$

where

$$
G_{0}^{\mathrm{FL}}=\frac{e^{2} \bar{\Gamma}^{2}}{\pi \hbar} V=2 \bar{\Gamma}^{2} \frac{e^{2}}{h}
$$

and

$$
G_{\mathrm{AB}}^{\mathrm{FL}}=-\frac{e^{2} \bar{\Gamma}^{2}}{\pi \hbar} \frac{T / T_{0}}{\sinh \left(T / T_{0}\right)}=-2 \bar{\Gamma}^{2} \frac{T / T_{0}}{\sinh \left(T / T_{0}\right)} \frac{e^{2}}{h} .
$$

The factor of 2 in the background term (14) comes from the two parallel tunneling paths in Fig. 2 b, each having transmission probability $\bar{\Gamma}^{2}$. The expressions (10) and (13) show that the line shape of the AB oscillations as a function of flux or $\mu$ is strictly sinusoidal, with a temperature-independent linewidth. Only the amplitude of the oscillations, given by $I_{\mathrm{AB}}^{\mathrm{FL}}$ and $G_{\mathrm{AB}}^{\mathrm{FL}}$, has a temperature dependence. Note that (13) also shows that the relevant Fourier component of the conductance oscillations has the same temperature dependence as $G_{\mathrm{AB}}^{\mathrm{FL}}$.

The calculation presented above is valid for a noninteracting electron system only, and therefore does not apply to FQHE edge states. Nonetheless, it is possible to extend these Fermi liquid results to the FQHE regime by assuming that the Büttiker-Landauer formula (5) and the transmission probability (7) can be applied to noninteracting composite fermions [40]. With this assumption the transport properties in the FQHE regime become qualitatively similar to that in the integer regime and to the existing antidot experiments [28,29]. The approach we take in this paper, however, is the microscopic one based on CLL theory.

\section{FINITE-SIZE CHIRAL LUTTINGER LIQUID WITH TOPOLOGICAL EXCITATIONS}

To study mesoscopic effects associated with edge states in the FQHE, we shall perform a quantization of CLL theory for a finite-size system and include the possibility of topological excitations of the chiral scalar field and coupling to an AB flux. Finite-size effects in nonchiral Luttinger liquids have been discussed previously by Haldane [9] and by Loss [31]. To proceed in the chiral case we bosonize the electron field operators $\psi_{ \pm}(x)$ according to the convention

$$
\rho_{ \pm}(x)= \pm \frac{\partial_{x} \phi_{ \pm}}{2 \pi}
$$

where

$$
\rho_{ \pm}(x) \equiv \lim _{a \rightarrow 0}: \psi_{ \pm}^{\dagger}(x+a) \psi_{ \pm}(x):
$$

is the normal-ordered charge density and $\phi_{ \pm}(x)$ is a chiral scalar field for right $(+)$ or left $(-)$ movers. The dynamics is governed by Wen's Euclidian action [10]

$$
S_{ \pm}=\frac{1}{4 \pi g} \int_{0}^{L} d x \int_{0}^{\beta} d \tau \partial_{x} \phi_{ \pm}\left( \pm i \partial_{\tau} \phi_{ \pm}+v \partial_{x} \phi_{ \pm}\right),
$$

where $g=1 / q$ (with $q$ odd) is the bulk filling factor, $v$ is the edge-state Fermi velocity (edge-magnetoplasmon velocity), and $L$ is the size of the edge state. When $q=1$, 
the action (18) describes noninteracting chiral electrons. The Lagrangian and real-time equations of motion are

$$
\mathcal{L}_{ \pm}=\frac{1}{4 \pi g} \partial_{x} \phi_{ \pm}\left(\mp \partial_{t} \phi_{ \pm}-v \partial_{x} \phi_{ \pm}\right),
$$

and

$$
\left(\partial_{x} \partial_{t} \pm v \partial_{x}^{2}\right) \phi_{ \pm}(x, t)=0 .
$$

The field theory described by (18) can be canonically quantized by imposing the equal-time commutation relation

$$
\left[\phi_{ \pm}(x), \phi_{ \pm}\left(x^{\prime}\right)\right]= \pm i \pi g \operatorname{sgn}\left(x-x^{\prime}\right) .
$$

Furthermore, the left and right sectors commute,

$$
\left[\phi_{-}(x), \phi_{+}\left(x^{\prime}\right)\right]=0 .
$$

The momentum density canonically conjugate to $\phi_{ \pm}(x)$ is therefore identified as $\mp \partial_{x} \phi_{ \pm} / 2 \pi g$. We then decompose $\phi_{ \pm}(x)$ into a nonzero-mode contribution $\phi_{ \pm}^{\mathrm{p}}(x)$ satisfying periodic boundary conditions that describes the neutral excitations, and a zero-mode part $\phi_{ \pm}^{0}(x)$ that contributes to the charged excitations,

$$
\phi_{ \pm}(x)=\phi_{ \pm}^{\mathrm{p}}(x)+\phi_{ \pm}^{0}(x) .
$$

The nonzero-mode part may be expanded in a basis of Bose annihilation and creation operators as

$$
\phi_{ \pm}^{\mathrm{p}}(x)=\sum_{k \neq 0} \theta( \pm k) \sqrt{\frac{2 \pi g}{|k| L}}\left(a_{k} e^{i k x}+a_{k}^{\dagger} e^{-i k x}\right) e^{-|k| a / 2},
$$

where $\left[a_{k}, a_{k^{\prime}}^{\dagger}\right]=\delta_{k k^{\prime}}$, with the coefficients in (24) determined by the requirement that $\phi_{ \pm}^{\mathrm{p}}(x)$ itself satisfies (21) in the $L \rightarrow \infty$ limit. In a finite-size system, however, it can be shown that

$$
\left[\phi_{ \pm}^{\mathrm{p}}(x), \phi_{ \pm}^{\mathrm{p}}\left(x^{\prime}\right)\right]= \pm i \pi g \operatorname{sgn}\left(x-x^{\prime}\right) \mp \frac{2 \pi i g\left(x-x^{\prime}\right)}{L},
$$

so we must require the zero-mode contribution to satisfy

$$
\left[\phi_{ \pm}^{0}(x), \phi_{ \pm}^{0}\left(x^{\prime}\right)\right]= \pm \frac{2 \pi i g\left(x-x^{\prime}\right)}{L}
$$

for the total field to satisfy (21). An expansion analogous to (24) for the zero-modes can be constructed from the condition (26) and, in addition, the requirement

$$
\phi_{ \pm}^{0}(x+L)-\phi_{ \pm}^{0}(x)= \pm 2 \pi N_{ \pm},
$$

which follows from (16), where

$$
N_{ \pm} \equiv \int_{0}^{L} d x \rho_{ \pm}(x)
$$

is the charge of an excited state relative to the ground state. Conditions (26) and (27) together determine $\phi_{ \pm}^{0}(x)$, up to an additive c-number constant, as

$$
\phi_{ \pm}^{0}(x)= \pm \frac{2 \pi}{L} N_{ \pm} x-g \chi_{ \pm},
$$

where $\chi_{ \pm}$is an Hermitian phase operator canonically conjugate to $N_{ \pm}$satisfying

$$
\left[\chi_{ \pm}, N_{ \pm}\right]=i \text {. }
$$

Equations (24) and (29) may now be used to write the normal-ordered CLL Hamiltonian as

$$
\begin{aligned}
H_{ \pm} & =\frac{v}{4 \pi g} \int_{0}^{L} d x\left(\partial_{x} \phi_{ \pm}\right)^{2} \\
& =\frac{\pi v}{g L} N_{ \pm}^{2}+\sum_{k} \theta( \pm k) v|k| a_{k}^{\dagger} a_{k} .
\end{aligned}
$$

The normal-ordered charge $N_{ \pm}$is a constant of the motion, whereas $\partial_{t} \chi_{ \pm}=2 \pi v N_{ \pm} / g L$. The normal-ordered density operator (16) for an isolated edge state satisfies the chiral equations of motion

$$
\left(\partial_{t} \pm v \partial_{x}\right) \rho_{ \pm}=0 .
$$

Also note that the compressibility $\kappa_{ \pm} \equiv \partial \rho_{ \pm} / \partial \mu$ of the uniform CLL is $\kappa_{ \pm}=g / 2 \pi v$, half the spinless nonchiral Luttinger liquid value. In a finite-size system, the level spacing for neutral and charged excitations is of the order of $v / L$, and both types of edge excitations become gapless in the $L \rightarrow \infty$ limit as expected.

We turn now to a discussion of the bosonization of the right (+) and left (-) moving components of the electron field operators. Eqn. (16) shows that to create an electron, we need to create a $\pm 2 \pi$ step in the chiral scalar field. The electron field operators can be bosonized as

$$
\psi_{ \pm}(x)=\frac{1}{\sqrt{2 \pi a}} e^{i\left[\phi_{ \pm}(x) \pm \frac{\pi x}{L}\right] / g},
$$

where $a$ is the same microscopic cutoff length that appears in (24). To see that (34) is valid, note that

$$
\left[\rho_{ \pm}(x), \psi_{ \pm}^{\dagger}\left(x^{\prime}\right)\right]=\delta\left(x-x^{\prime}\right) \psi_{ \pm}^{\dagger}\left(x^{\prime}\right),
$$

so $\psi_{ \pm}^{\dagger}(x)$ creates an electron at position $x$. The necessary time-dependent generalization of (34) is

$$
\psi_{ \pm}(x, t)=\frac{1}{\sqrt{2 \pi a}} e^{i \phi_{ \pm}(x, t) / g} e^{ \pm i \pi(x \mp v t) / g L},
$$

where $\phi_{ \pm}(x, t)$ is in the Heisenberg representation.

It is important to realize that the additional c-number phase factor $e^{ \pm i \pi x / g L}$, which has the effect of disentangeling the charge and phase operators in the zero-mode, is necessary for bosonizaton in a finite-size system. An example of this necessity is provided by a calculation of the equal-time correlation function 


$$
C_{ \pm}(x) \equiv\left\langle\psi_{ \pm}(x) \psi_{ \pm}^{\dagger}(0)\right\rangle
$$

for the finite-size chiral Fermi liquid $(g=1)$ at zero temperature, which can be calculated via bosonization and also directly. Using (34) we find

$$
\begin{aligned}
C_{ \pm}(x) & =\frac{e^{ \pm i \pi x / L}}{2 \pi a}\left\langle e^{i\left(\phi_{ \pm}^{0}(x)-\phi_{ \pm}^{0}(0)\right)}\right\rangle \\
& \times e^{\frac{1}{2}\left[\phi_{ \pm}^{0}(x), \phi_{ \pm}^{0}(0)\right]} e^{\left\langle\phi_{ \pm}^{\mathrm{p}}(x) \phi_{ \pm}^{\mathrm{p}}(0)-\left(\phi_{ \pm}^{\mathrm{p}}(0)\right)^{2}\right\rangle} .
\end{aligned}
$$

At zero temperature,

$$
\left\langle e^{i\left(\phi_{ \pm}^{0}(x)-\phi_{ \pm}^{0}(0)\right)}\right\rangle=\left\langle e^{ \pm 2 \pi i N_{ \pm} x / L}\right\rangle=1
$$

and (for $g=1$ )

$$
\left\langle\phi_{ \pm}^{\mathrm{p}}(x) \phi_{ \pm}^{\mathrm{p}}(0)-\left(\phi_{ \pm}^{\mathrm{p}}(0)\right)^{2}\right\rangle=\mathcal{S}( \pm x),
$$

where $\mathcal{S}(x)$ is defined in Appendix B. Thus, we find

$$
C_{ \pm}(x)=\frac{( \pm i / 2 L) e^{ \pm i \pi x / L}}{\sin [\pi(x \pm i a) / L]} .
$$

Note that (41) has the correct periodicity $C_{ \pm}(x+L)=$ $C_{ \pm}(x)$. The same quantity (37) may also be calculated directly from the chiral Fermi liquid Hamiltonian

$$
H_{ \pm}=\sum_{k} \epsilon_{ \pm}(k): c_{ \pm}^{\dagger}(k) c_{ \pm}(k):
$$

normal-ordered with respect to an infinite Dirac sea as in the Luttinger model. Here $c_{ \pm}^{\dagger}(k)$ and $c_{ \pm}(k)$ denote creation and annihilation operators for the right $(+)$ or left $(-)$ branch, $\epsilon_{ \pm}(k)= \pm v k$ are the energies of the infinite linear branches, and $\psi_{ \pm}(x)=L^{-\frac{1}{2}} \sum_{k} e^{i k x} c_{ \pm}(k)$. We then obtain

$$
C_{ \pm}(x)=\frac{1}{L} \sum_{k} e^{i k x}\left\langle 1-c_{ \pm}^{\dagger}(k) c_{ \pm}(k)\right\rangle .
$$

The ground state momentum distribution function $n_{ \pm}(k)$ is given by $n_{+}(k)=1$ for $k \leq 0, n_{-}(k)=1$ for $k \geq 0$, and $n_{ \pm}(k)=0$ elsewhere. [This may be written as $n_{ \pm}(k)=\theta(\mp k)$ with the understanding that the full $k=0$ state is to be included.] Then we find

$$
C_{ \pm}(x)=\frac{1}{L} \sum_{k>0} e^{ \pm i k x} e^{-k a}
$$

where we have included a convergence factor. This result is identical to the form (41) calculated with the finite-size bosonization formula (34).

The bosonization formula (34) may also be used to determine the allowed eigenvalues of the charge operator $N_{ \pm}$. Equation (34) implies that

$$
\psi_{ \pm}^{\dagger}(x+L)=\psi_{ \pm}^{\dagger}(x) e^{\mp 2 \pi i N_{ \pm} / g}
$$

Thus, periodic boundary conditions on the electron creation operators lead to the result that the allowed eigenvalues of $N_{ \pm}$are given by

$$
N_{ \pm}=n g
$$

where $n$ is any integer, which means that there exists fractionally charged excitations, as expected in a FQHE system. The result (46) also follows from the requirement that the annihilation operators $\psi_{ \pm}(x)$ satisfy periodic boundary conditions.

The CLL theory (18) is valid for any magnetic field strength within a given fractional plateau (of the form $1 / q$ with $q$ odd) that makes the bulk quantum Hall fluid incompressible, but does not distinguish between these different possible magnetic fields. This is because the action (18) does not know whether the edge it describes is at the boundary of a Hall bar, where the precise location within a Hall plateau is unimportant, or is at the boundary of a quantum Hall droplet or an antidot piercing an otherwise uniform fluid, where there are additional mesoscopic effects associated with the actual position within the plateau. To account for these mesoscopic effects in the antidot system, we couple to an $\mathrm{AB}$ flux $\Phi$ by adding a term

$$
\delta \mathcal{L}_{ \pm}=\frac{1}{c} j_{ \pm} A
$$

to the CLL Lagrangian (19) where $j_{ \pm}(x)$ is the onedimensional current density associated with $\rho_{ \pm}(x)$, as defined through the continuity equation $-e \partial_{t} \rho_{ \pm}+\partial_{x} j_{ \pm}=0$. Using (33) we obtain a bosonized expression for the current density, $j_{ \pm}= \pm \frac{e}{2 \pi} \partial_{t} \phi_{ \pm}$. The vector potential in (47) is given by $A=\Phi / L$. The flux couples only to the zero-modes, and results in the Hamiltonian

$$
H_{ \pm}=\frac{\pi v}{g L}\left(N_{ \pm} \pm g \varphi\right)^{2}+\sum_{k} \theta( \pm k) v|k| a_{k}^{\dagger} a_{k}
$$

where $\varphi \equiv \Phi / \Phi_{0}$ is the dimensionless flux.

\section{SCALING THEORY}

In Section II we studied the AB effect in the integral quantum Hall regime, where the edge states are chiral Fermi liquids, by using the Büttiker-Landauer formula, which is valid for all values of the antidot tunneling amplitudes. In the fractional regime, where the edge states are CLLs, the strong electron correlation dramatically changes the physics of the tunneling process itself, as emphasized by Wen 10 and also by Kane and Fisher 111 and Matveev et al. 42 in the context of the onedimensional interacting electron gas. Whereas electron tunneling between FQHE edge states is inhibited because an electron added to an edge state is not properly correlated with the others already there, tunneling of fractionally charged quasiparticles, when allowed, is actually enhanced and may become divergent at low temperatures. 
Quasiparticle tunneling is difficult to treat theoretically because of this nonperturbative aspect.

The renormalization group ( $\mathrm{RG}$ ) has played a central role in the theory of the chiral and nonchiral Luttinger liquids, and we now briefly review its connection with the divergent quasiparticle tunneling between FQHE edge states in a quantum point contact. The divergence reflects the fact that the quasiparticle tunneling operator is relevant in the RG sense 11, 13, and in the zero-temperature limit CLL theory predicts that in the quantum-point-contact geometry the Hall fluid with interedge tunneling is an insulator. If, by adjusting the magnetic field or a gate voltage or both, a condition of complete destructive interference is achieved that prevents the aforementioned tunneling, a resonance peak in the two-terminal conductance will occur [43]. However, this on-resonance fixed-point is clearly unstable and the system will try to flow to the stable insulating fixedpoint. The situation is entirely analogous to a secondorder phase transition, and here too we expect critical exponents associated with the unstable fixed-point. These exponents, which reflect the form of the tunneling conductance near a resonance, can be obtained from a RG analysis. Because no other operators allowed by symmetry are relevant at low temperatures, Kane and Fisher [11] went further by proposing that the entire RG trajectory from Hall conductor to insulator will be universal, resulting in a universal lineshape near and away from resonance. The universal lineshape for the $g=1 / 3$ case has been calculated exactly by Fendley, Ludwig, and Saleur [17] using the thermodynamic Bethe ansatz.

The scaling behavior in the antidot geometry differs in two important ways. First, although the RG flow equations are the same here as in the quantum-point-contact geometry, their physical implications are different. Recall that in the quantum-point-contact system it is assumed, and reasonably so, that there exist conditions of complete destructive interference that will cause a resonance. But in the antidot system the origin of interference is the AB effect, which, because of the chirality, never leads to the necessary complete destructive interference for any finite antidot-coupling strength (see the discussions in Section I and Appendix A). The two-terminal conductance on resonance, $G^{*}$, is determined by the antidot-coupling strength and the temperature and is always less than $g e^{2} / h$. The second important difference between the two geometries is the role of the additional scale $T_{0}$ in the antidot case. Whereas in the quantum-point-contact geometry the universal RG trajectory implies a one-parameter universal scaling function $\tilde{G}(X)$, where $X$ depends on the temperature, the $R G$ trajectory in the antidot problem, which we also predict to be universal for sufficiently low $T_{0}$, leads instead to a two-parameter universal scaling function $\tilde{G}(X, Y)$, where $Y$ depends on the size of the antidot and is temperature independent. The scaling function $\tilde{G}(X, Y)$ contains all the mesoscopic effects associated with the finite-size antidot edge state.

We turn now to a detailed RG theory of the antidot problem. We begin by performing a perturbative analysis in the weak-antidot-coupling regime shown schematically in Fig. 2a. In this case we have

$$
S=S_{0}+\delta S
$$

where $S_{0} \equiv S_{\mathrm{L}}+S_{\mathrm{R}}+S_{\mathrm{A}}$ is the sum of actions of the form (18) for the left moving, right moving, and antidot edge states, respectively, and

$$
\delta S \equiv \sum_{m=1}^{\infty} \int_{0}^{\beta} d \tau\left(V_{+}(\tau)+V_{-}(\tau)+\text { c.c. }\right)
$$

is the weak coupling between them. Here

$$
V_{ \pm}(\tau) \equiv \frac{v \Gamma_{ \pm}^{(m)}}{2 \pi a} e^{i m \phi_{ \pm}\left(x_{ \pm}, \tau\right)} e^{-i m \phi_{A}\left(x_{ \pm}, \tau\right)}
$$

describes the tunneling of $m$ quasiparticles from an incident edge state into the antidot edge state at point $x_{ \pm}$with dimensionless amplitude $\Gamma_{ \pm}^{(m)}$ 11. In the weakantidot-coupling regime $\left|\Gamma_{ \pm}^{(m)}\right| \ll 1$. The form of the tunneling perturbation (51) follows from a generalization of the bosonization formula (34) to particles with fractional charge and fractional statistics; the edge-state velocity factor $v$ is included to make the $\Gamma_{ \pm}^{(m)}$ dimensionless. The phase factor in (34) is unnecessary here and for simplicity will be omitted. Because the high-energy cutoff of the theory - the effective Fermi energy - is of order $v / a$, the magnitudes of the $\Gamma_{ \pm}^{(m)}$ are essentially tunneling rates in units of the Fermi energy. (Recall that $a$ is a microscopic cutoff length taken to be of the order of the magnetic length.) We assume the incident edge states or leads, described by $S_{\mathrm{L}}$ and $S_{\mathrm{R}}$, to be macroscopic, and we also assume for simplicity that $\left|\Gamma_{-}^{(m)}\right|=\left|\Gamma_{+}^{(m)}\right|$. Furthermore, it is not necessary to explicitly include the flux in $S_{\mathrm{A}}$ so we set $\varphi=0$.

It is most convenient to perform the RG analysis directly in terms of the Euclidian action (49), and hence in this section expectation values of fields refer to their functional-integral form 44,

$$
\left\langle\phi_{ \pm}\left(x_{1}, \tau_{1}\right) \phi_{ \pm}\left(x_{2}, \tau_{2}\right) \cdots\right\rangle=\frac{1}{Z_{ \pm}} \int \mathcal{D} \phi_{ \pm} \phi_{ \pm}\left(x_{1}, \tau_{1}\right) \phi_{ \pm}\left(x_{2}, \tau_{2}\right) \cdots e^{-S_{ \pm}}
$$

where $Z_{ \pm} \equiv \int \mathcal{D} \phi_{ \pm} e^{-S_{ \pm}}$is the edge-state partition function. The allowed field configurations in (52) satisfy the boundary conditions

$$
\begin{aligned}
& \phi_{ \pm}(x+L, \tau)-\phi_{ \pm}(x, \tau)=2 \pi n g \\
& \phi_{ \pm}(x, \tau+\beta)-\phi_{ \pm}(x, \tau)=0
\end{aligned}
$$


and the measure in (52) implicitly includes a sum over the integer winding number $n$. Note that there is only one homotopy index here, because in the CLL a topological charge excitation and topological current excitation are equivalent.

For our analysis we will need the Euclidian $m$-quasiparticle propagator

$$
G_{ \pm}^{(m)}(x, \tau) \equiv\left\langle e^{i m \phi_{ \pm}(x, \tau)} e^{-i m \phi_{ \pm}(0)}\right\rangle
$$

for a right $(+)$ or left $(-)$ moving edge state, which at zero temperature is given by

$$
G_{ \pm}^{(m)}(x, \tau)=\left(\frac{ \pm i \pi a / L}{\sin [\pi(x \pm i v \tau) / L]}\right)^{m^{2} g}
$$

where we have neglected the regularization in the denominator, which is only necessary when $x \pm i v \tau=0$. The expression (56) is calculated in Appendix B using the finite-size bosonization formalism developed in Section III. Note that when $x=0$, the largest contribution to an integral of (56) over imaginary time comes from small $\tau$ where we have $G_{ \pm}^{(m)}(0, \tau) \sim(1 / \tau)^{2 \Delta}$, where $\Delta=m^{2} g / 2$ is the local scaling dimension of $e^{i m \phi_{ \pm}}$.

Consider now the correlation function

$$
\begin{aligned}
\left\langle V_{+}(\tau) V_{+}^{*}(0)\right\rangle & =\frac{v^{2}\left|\Gamma_{+}^{(m)}\right|^{2}}{4 \pi^{2} a^{2}}\left\langle e^{i m \phi_{+}\left(x_{+}, \tau\right)} e^{-i m \phi_{+}\left(x_{+}, 0\right)}\right\rangle\left\langle e^{-i m \phi_{A}\left(x_{+}, \tau\right)} e^{i m \phi_{A}\left(x_{+}, 0\right)}\right\rangle \\
& =\frac{v^{2}\left|\Gamma_{+}^{(m)}\right|^{2}}{4 \pi^{2} a^{2}} G_{+}^{(m)}(0, \tau) G_{\mathrm{A}}^{(m)}(0,-\tau)
\end{aligned}
$$

which arises in a perturbative calculation of the full partition function

$$
Z=\int \mathcal{D} \phi_{L} \mathcal{D} \phi_{R} \mathcal{D} \phi_{A} e^{-S}
$$

at second order. For $\left\langle V_{+}(\tau) V_{+}^{*}(0)\right\rangle$-and therefore $Z$ to be invariant under a small increase in unit-cell size $a \rightarrow a^{\prime}=b a$, we require $\Gamma_{+}^{\prime}=b^{1-2 \Delta} \Gamma_{+}$or $d \Gamma_{+} / d \ln b=$ $\left(1-m^{2} g\right) \Gamma_{+}$. An analogous analysis of $\left\langle V_{-}(\tau) V_{-}^{*}(0)\right\rangle$ shows that $\Gamma_{-}$scales identically. These leading-order flow equations,

$$
\frac{d \Gamma_{ \pm}^{(m)}}{d \ell}=\left(1-m^{2} g\right) \Gamma_{ \pm}^{(m)}
$$

where $\ell \equiv \ln \left(a^{\prime} / a\right)$, show that quasiparticle $(m=1)$ backscattering processes are relevant whereas electron $(m=1 / g)$ backscattering is irrelevant when $g=1 / 3$, as stated above. They were first derived by Kane and Fisher [1] using momentum-shell RG.

One might expect the flow equations (59) to be modified by the finite-size of the antidot edge state. To see that this is not so, consider the correlation function

$$
\left\langle V_{+}(\tau) V_{+}^{*}\left(\tau^{\prime}\right) V_{-}\left(\tau^{\prime \prime}\right) V_{-}^{*}(0)\right\rangle,
$$

which appears at fourth order in $\delta S$. A Wick expansion gives local terms as in (57), and, in addition, nonlocal antidot propagators $G_{\mathrm{A}}^{(m)}(x, \tau)$ with $x=L / 2$, where $L$ is now the circumference of the antidot edge state. However, the expression (56) shows that nonlocal terms scale in the same way as the local terms. The simplicity of this conclusion is the advantage of our RG method: It focuses directly on the scaling of the cutoff $a$ rather than on the scaling of $\tau$, which is inconvenient when $x \neq 0$. Our conclusion is that the Kane-Fisher flow equations (59) are valid in the antidot geometry considered here.

Exactly on resonance, defined by the condition that all the $\Gamma_{ \pm}^{(m)}$ are zero, we have perfect source-drain transmission with $G^{*}=g e^{2} / h$. Note, however, that this perfect resonance can be experimentally realized only by physically decoupling the antidot from the leads - it cannot occur because of the AB effect itself. Nonetheless, the perfect resonance is still a fixed point solution of (59), albeit a physically uninteresting one. However, in the weak-antidot-coupling regime of Fig. $2 \mathrm{a}$, the bare tunneling amplitudes can be very small, in which case the leading-order RG equations (59) yield

$$
\Gamma_{ \pm, \mathrm{ren}}^{(m)}=\left(\frac{a_{\mathrm{ren}}}{a}\right)^{1-m^{2} g} \Gamma_{ \pm}^{(m)} .
$$

Here $\Gamma_{ \pm}^{(m)}$ are the bare tunneling amplitudes and $\Gamma_{ \pm, \text {ren }}^{(m)}$ are the renormalized coupling constants at a length scale $a_{\text {ren }}>a$. In the $g=1 / 3$ case we see that the renormalized $m=1$ quasiparticle tunneling amplitude diverges as $\left(a_{\text {ren }} / a\right)^{2 / 3}$, whereas all others with $m>1$ vanish as $\left(a_{\text {ren }} / a\right)^{1-m^{2} / 3}$. However, even at zero temperature in an infinite Hall bar, the scaling may be cut off by the finite size $L$ of the antidot edge state, because the effective unit-cell size $a_{\text {ren }}$ cannot become larger than the antidot 45. Thus, the renormalized $m=1$ quasiparticle tunneling amplitude will not diverge here, but it can still become very large, of the order of $(L / a)^{2 / 3}$ times the bare value, where $L / a$ is typically $10^{2}$. At finite temperature the thermal length $L_{\mathrm{T}}=v / T$, which is the size of the system in the imaginary-time direction, will also cutoff 
the scaling behavior. The maximum allowed $a_{\text {ren }}$ is the minimum $L_{\min }$ of $L$ and $L_{\mathrm{T}}$, so the final renormalized couplings in the weak-antidot-coupling regime are

$$
\Gamma_{ \pm, \text {ren }}^{(m)}=\left(L_{\min } / a\right)^{1-m^{2} g} \Gamma_{ \pm}^{(m)} .
$$

At temperatures less than the crossover temperature $T_{0}$, defined in Eqn. (11), we see that the renormalized tunneling amplitudes are determined by $L$ whereas above $T_{0}$ they are determined by $L_{\mathrm{T}}$.

As in the conventional momentum-shell procedure, our RG transformation is based on a coarse graining of the system followed by a rescaling to hold the partition function (58) fixed. The flow equations (59) therefore hold quite generally for equilibrium and nonequilibrium properties, but, for this same reason, do not directly describe the scaling of the specific quantity we are after, namely, the two-terminal linear conductance as a function of flux. [In fact, including the $\mathrm{AB}$ flux $\varphi$ in the antidot action $S_{\mathrm{A}}$ simply modifies the propagator $G_{\mathrm{A}}^{(m)}(x, \tau)$ by a phase and does not change the RG equations.] To obtain scaling equations specific to the conductance $G$ as a function of tunneling amplitudes, temperature, and flux, one should perform the RG transformation keeping $G$ itself invariant. However, near a resonance the effect of the flux on the quantity $G$ is to simply vary the tunneling amplitudes $\Gamma_{ \pm}^{(m)}$. Therefore, in our application of (59) and (62) to the study of $G$, we can simply regard the $\Gamma_{ \pm}^{(m)}$ as being fluxdependent and gate-voltage dependent quantities, as in the quantum-point-contact geometry. This can be made precise by the introduction of a resonance tuning parameter $\delta$, which is a function of $\mathrm{AB}$ flux or gate voltage or both, and which specifies the distance from a perfect resonance $\delta=0$ in these units. Note, however, that we cannot set $\delta$ equal to zero, because there are no perfect resonances here.

We are now in a position to understand the nature of the resonances in the antidot geometry at temperatures low enough where all tunneling amplitudes except for the $\Gamma_{ \pm}^{(1)}$ have scaled to negligible values. The precise temperature $T_{\mathrm{s}}$ below which this scaling occurs depends on the values of the bare irrelevant couplings, $\Gamma_{ \pm}^{(m)}$ (with $m>1$ ), and, of course, how small one requires them to be. We shall also assume that $T_{0}<T_{\mathrm{s}}$, which is physically realizable in an antidot that is not too small. When $T$ and $T_{0}$ are both less than $T_{\mathrm{s}}$ the RG flow will follow a univeral trajectory, as in the quantum-point-contact problem [13]. Then there is a single correlation length $\xi$ that diverges on resonance $\Gamma_{ \pm}^{(1)}=0$ with a critical exponent determined by (59),

$$
\xi \sim\left(\frac{1}{\left|\Gamma_{ \pm}^{(1)}\right|}\right)^{\frac{1}{1-g}}
$$

and the conductance as a function of $\left|\Gamma_{ \pm}^{(1)}\right| \propto \delta$, the thermal length $L_{\mathrm{T}}$, and the antidot size $L$ will obey the scaling law

$$
\begin{aligned}
G\left(\delta, L_{\mathrm{T}}, L\right) & =\tilde{G}^{\prime}\left(c_{1} \frac{L_{\mathrm{T}}}{\xi}, c_{2} \frac{L}{\xi}\right) \\
& =\tilde{G}\left(c_{1} \frac{\delta}{T^{1-g}}, c_{2} \frac{\delta}{T_{0}^{1-g}}\right),
\end{aligned}
$$

where $c_{1}$ and $c_{2}$ are nonuniversal constants and $\tilde{G}(X, Y)$ is a two-parameter universal scaling function. In the second form in (64) we have expressed the antidot size in terms of the crossover temperature $T_{0}$ using (1). Near the resonance, (64) expresses the conventional assumption about finite-size scaling near a critical point, in both the real-space and imaginary time directions. Much less trivial is our assumption, following a similar one by Kane and Fisher [1], that (64) holds for all values of $\delta$ over which $\left|\Gamma_{ \pm}^{(1)}\right| \propto \delta$. The universal scaling function $\tilde{G}(X, Y)$ will be valid as long as the temperature is low enough so that the corrections to scaling from the irrelevant operators are small and as long as the resonances are narrow enough so that the linear relation $\left|\Gamma_{ \pm}^{(1)}\right| \propto \delta$ applies.

In the limit $L \rightarrow a$ the antidot system becomes equivalent to quantum point contact with a single miscoscopic impurity providing the momentum transfer to the lattice necessary for tunneling. The crossover from CLL powerlaw behavior to nearly Fermi-liquid-like scaling caused by mesoscopic effects that we discuss in detail in the next section does not occur in this limit because $T_{0}$ is pushed up to the high-energy cutoff $T_{\mathrm{F}} \equiv v / a$, the effective Fermi temperature, beyond which CLL is invalid and the FQHE does not occur. Therefore in the $Y \rightarrow 0$ limit our two-parameter scaling function $\tilde{G}(X, Y)$ reduces to the one-parameter function $\tilde{G}(X)$ defined by Kane and Fisher 11 and calculated by Moon et al. 13 and by Fendley, Ludwig, and Saleur [17]:

$$
\tilde{G}(X, 0)=\tilde{G}(X) .
$$

An explicit form for $\tilde{G}(X, Y)$, valid for $X \gg 1$, may be obtained from perturbation theory in the strong-antidotcoupling regime, but will not be needed here.

The scaling law (64) shows that there is a temperature scale $T_{1}$ determined by the bare quasiparticle tunneling amplitude $\Gamma_{ \pm}^{(1)}$ below which the system is always in the strong-antidot-coupling regime. This means that the resonances are never perfect, and at temperatures less than $T_{1}$ the on-resonance conductance $G^{*} \ll g e^{2} / h$. At temperatures above $T_{1}$ a weak-antidot-coupling regime is of course possible for $\left|\Gamma_{ \pm}^{(m)}\right| \ll 1$, and in this case

$$
G^{*}=\left[1-\mathcal{O}\left(\Gamma^{4}\right)\right] g \frac{e^{2}}{h} \approx g \frac{e^{2}}{h} .
$$

If the antidot system starts in the strongly coupled regime, by an appropriate choice of gate voltages, it will stay in this regime throughout the experimentally relevant ranges of temperature and magnetic field, because the $m=1$ quasiparticle backscattering process (which would be relevant in the RG sense) is not allowed in 
this edge-state configuration and only electrons can tunnel 46]. The strong-antidot-coupling regime therefore admits a perturbative treatment [46], to which we now turn.

\section{AHARONOV-BOHM EFFECT IN THE STRONG-ANTIDOT-COUPLING REGIME}

The current $I$ passing between edge states $L^{\prime}$ and $R^{\prime}$ as a function of their potential difference $V$ may be calculated for small energy-independent tunneling amplitudes $\Gamma_{i}(i=1,2)$, which for simplicity are taken to be equal apart from dynamical and AB phase factors,

$$
\begin{aligned}
& \Gamma_{1}=\Gamma e^{i \pi\left(\frac{\mu}{\Delta \epsilon}+\varphi\right)} \\
& \Gamma_{2}=\Gamma e^{-i \pi\left(\frac{\mu}{\Delta \epsilon}+\varphi\right)} .
\end{aligned}
$$

Here $\varphi \equiv \Phi / \Phi_{0}$, where $\Phi_{0} \equiv h c / e$ is the flux quantum, and $\mu \equiv\left(\mu_{\mathrm{L}}+\mu_{\mathrm{R}}\right) / 2$ is the mean electrochemical potential. These phases account for the total phase $\theta \equiv \oint_{\epsilon} d \mathbf{l} \cdot\left(\mathbf{p}+\frac{e}{c} \mathbf{A}\right)$ accumulated by an electron with energy $\mu$ after one complete clockwise orbit around the antidot. The Hamiltonian is $H=H_{0}+\delta H$, where
$H_{0}=H_{\mathrm{L}}+H_{\mathrm{R}}$ is a sum of Hamiltonians of the form (32) and

$$
\delta H=\Gamma_{1} B_{1}+\Gamma_{2} B_{2}+\Gamma_{1}^{*} B_{1}^{\dagger}+\Gamma_{2}^{*} B_{2}^{\dagger},
$$

where $B_{i} \equiv \psi_{L}\left(x_{i}\right) \psi_{R}^{\dagger}\left(x_{i}\right)$ is an electron tunneling operator acting at point $x_{i}$. Because the edge states $L^{\prime}$ and $R^{\prime}$ are assumed to be infinite, the additional c-number phase factor in (34) is not needed here. The current $I(t) \equiv-e\left\langle\dot{N}_{\mathrm{L}}\right\rangle$ to first order in $\delta H$ is given by

$$
I(t)=i e \int d t^{\prime} \theta\left(t-t^{\prime}\right) \operatorname{Tr} \rho_{0}\left[\partial_{t} \tilde{N}_{\mathrm{L}}(t), \delta \tilde{H}\left(t^{\prime}\right)\right]
$$

where $\rho_{0} \equiv e^{-\beta K_{0}} / \operatorname{Tr} e^{-\beta K_{0}}$,

$$
K_{0} \equiv H_{0}-\mu_{\mathrm{L}} N_{\mathrm{L}}-\mu_{\mathrm{R}} N_{\mathrm{R}}
$$

and where $(69)$ is written in the usual interaction representation $\tilde{O}(t) \equiv e^{i H_{0} t} O e^{-i H_{0} t}$. It is convenient, however, to work in the $K_{0}$-representation defined by $O(t) \equiv e^{i K_{0} t} O e^{-i K_{0} t}$. We find $\tilde{B}_{i}(t)=B_{i}(t) e^{i V t}$, where $V \equiv\left(\mu_{\mathrm{R}}-\mu_{\mathrm{L}}\right) / e$ is the applied voltage. Then (69) leads to

$$
I=-2|\Gamma|^{2} \operatorname{Im}\left[X_{11}(\omega)+X_{22}(\omega)+e^{2 \pi i\left(\frac{\mu}{\Delta \epsilon}+\varphi\right)} X_{12}(\omega)+e^{-2 \pi i\left(\frac{\mu}{\Delta \epsilon}+\varphi\right)} X_{21}(\omega)\right]_{\omega=e V},
$$

where $X_{i j}(\omega)$ is the Fourier transform of

$$
X_{i j}(t) \equiv-i \theta(t)\left\langle\left[B_{i}(t), B_{j}^{\dagger}(0)\right]\right\rangle .
$$

This response function can be calculated using bosonization techniques and the result for filling factor $g=1 / q$ is

$$
X_{i j}(t)=-\theta(t) \frac{a^{2 q-2}}{2 \pi^{2}} \operatorname{Im} \frac{\left(\pi / L_{\mathrm{T}}\right)^{2 q}}{\sinh ^{q}\left[\pi\left(x_{i}-x_{j}+v t+i a\right) / L_{\mathrm{T}}\right] \sinh ^{q}\left[\pi\left(x_{i}-x_{j}-v t-i a\right) / L_{\mathrm{T}}\right]},
$$

where $L_{\mathrm{T}} \equiv v / T$ is the thermal length. When $q=1,(73)$ is the response function for noninteracting chiral electrons. From (73) we see that $X_{11}=X_{22}$ and $X_{12}=X_{21}$, so (71) may be written as

$$
I=-4|\Gamma|^{2} \operatorname{Im}\left[X_{11}(\omega)+\cos \left[2 \pi\left(\frac{\mu}{\Delta \epsilon}+\varphi\right)\right] X_{12}(\omega)\right]_{\omega=e V} .
$$

Thus it is sufficient to calculate the imaginary part of $X_{i j}(\omega)$, which we shall do below.

Each term $X_{i j}$ in (71) corresponds to a process occurring with a probability proportional to $\left|\Gamma_{i} \Gamma_{j}\right|$. The local terms $X_{11}$ and $X_{22}$ therefore describe independent tunneling at $x_{1}$ and $x_{2}$, respectively, whereas the nonlocal terms $X_{12}$ and $X_{21}$ describe coherent tunneling through both antidot constrictions. The AB phase naturally couples only to the latter. We shall see that the local contributions behave exactly like the tunneling current in a quantum point contact. The $\mathrm{AB}$ effect, however, is a consequence of the nonlocal terms, and we shall show that there are new non-Fermi-liquid phenomena associated with these terms that are directly accessible to experiment.

The required Fourier transform may be calculated by contour integration. Here we shall present the calculation for the case $g=1 / 3$ (the other cases follow similarly). Because the factor multiplying $\theta(t)$ in $(73)$ is odd under $t \rightarrow-t$, the imaginary part of $X_{i j}(\omega)$ may be written as

$$
\operatorname{Im} X_{i j}(\omega)=-\frac{a^{4} \pi^{3} T^{5}}{8 v^{6}} \operatorname{Re} \int_{-\infty}^{\infty} d s \frac{e^{i \omega s / \pi T}-e^{-i \omega s / \pi T}}{\sinh ^{3}\left(s+\pi d / L_{\mathrm{T}}+i \eta\right) \sinh ^{3}\left(s-\pi d / L_{\mathrm{T}}+i \eta\right)},
$$

where $d \equiv\left|x_{i}-x_{j}\right|$ and $\eta$ is a positive infinitesimal. The local response functions $X_{11}$ and $X_{22}$ correspond to $d=0$, whereas the nonlocal ones $X_{12}$ and $X_{21}$ correspond to $d=L / 2$, where $L$ is the circumference of the antidot edge state. When $d \neq 0$ there are 3rd order poles at 


$$
s= \pm \frac{\pi d}{L_{\mathrm{T}}}+i n \pi-i \eta
$$

where $n$ is an integer. The integral of the first and second term in $(75)$ can be calculated by closing the integration contour in the upper-half-plane and lower-half-plane, respectively. One can show that the contributions from all the poles except $n=0$ cancel, leaving

$$
\begin{aligned}
\operatorname{Im} X_{i j}(\omega)=\frac{a^{4} \pi^{2}}{8 v^{6}} \frac{T^{3}}{\sinh ^{3}\left(2 \pi d / L_{\mathrm{T}}\right)}\{ & {\left[V^{2}+4 \pi^{2} T^{2}\left(1-3 \operatorname{coth}^{2}\left(2 \pi d / L_{\mathrm{T}}\right)\right)\right] \sin \left(\frac{V d}{v}\right) } \\
& \left.+6 \pi V T \operatorname{coth}\left(2 \pi d / L_{\mathrm{T}}\right) \cos \left(\frac{V d}{v}\right)\right\} .
\end{aligned}
$$

The case where $d=0$ may then be obtained by taking the $d \rightarrow 0$ limit of this expression. In the $g=1 / 3$ case one has to expand up to third order in $d$ (all lower orders cancel exactly).

The response function (77) evidently displays a crossover behavior when the thermal length $L_{T}$ becomes less than $\left|x_{i}-x_{j}\right|$. The finite size of the antidot therefore provides an important temperature scale (1D). For example, a Fermi velocity $v$ of $10^{6} \mathrm{~cm} / \mathrm{s}$ and circumference $L$ of $1 \mu \mathrm{m}$ yields $T_{0} \approx 25 \mathrm{mK}$. Note that $T_{0}$ is closely related to the energy level spacing $\Delta \epsilon$ for noninteracting electrons with linear dispersion in a ring of circumference $L$,

$$
T_{0}=\frac{\Delta \epsilon}{2 \pi^{2}}
$$

The current can generally be written as

$$
I=I_{0}+I_{\mathrm{AB}} \cos \left[2 \pi\left(\frac{\mu}{\Delta \epsilon}+\varphi\right)\right]
$$

where $I_{0}$ is the "background" current resulting from the local terms and $I_{\mathrm{AB}}$ is the $\mathrm{AB}$ current resulting from the nonlocal terms. When the voltage is applied symmetrically about an antidot energy level, $\cos \left[2 \pi\left(\frac{\mu}{\Delta \epsilon}+\varphi\right)\right]=\cos (2 \pi \varphi)$.

The exact current-voltage relation for the $g=1 / 3$ chiral Luttinger liquid is

$$
I_{0}=\frac{|\Gamma|^{2} a^{4}}{120 \pi v^{6}}\left(64 \pi^{4} V T^{4}+20 \pi^{2} T^{2} V^{3}+V^{5}\right),
$$

and

$$
I_{\mathrm{AB}}=-\frac{|\Gamma|^{2} a^{4} \pi^{2}}{v^{6}} \frac{T^{3}}{\sinh ^{3}\left(T / T_{0}\right)}\left\{\left[V^{2}+4 \pi^{2} T^{2}\left(1-3 \operatorname{coth}^{2}\left(T / T_{0}\right)\right)\right] \sin \left(\frac{V L}{2 v}\right)+6 \pi V T \operatorname{coth}\left(T / T_{0}\right) \cos \left(\frac{V L}{2 v}\right)\right\} .
$$

In the limit $L \rightarrow 0, I_{\mathrm{AB}}$ always reduces to $I_{0}$. The $\sin (V L / 2 v)$ and $\cos (V L / 2 v)$ factors have a period in $V$ equal to twice the level spacing $\Delta \epsilon$, as expected (see Section II). The AB conductance for $q=3$ is

$$
G_{\mathrm{AB}}=-\frac{2 \pi^{3}|\Gamma|^{2} a^{4}}{v^{6}} \frac{T^{4}}{\sinh ^{3}\left(T / T_{0}\right)}\left\{3 \operatorname{coth}\left(\frac{T}{T_{0}}\right)+\left(\frac{T}{T_{0}}\right)\left[1-3 \operatorname{coth}^{2}\left(\frac{T}{T_{0}}\right)\right]\right\}
$$

which is shown in Fig. A along with the corresponding chiral Fermi liquid result (15).

We now summarize our results for general $q$. We shall for convenience summarize the transport properties as a function of temperature for fixed voltage, first for $V \ll T_{0}$ and then for $V \gg T_{0}$.

\section{A. Low voltage regime: $V \ll T_{0}$}

There are three temperature regimes here. When $T \ll V \ll T_{0}$, both $I_{0}$ and $I_{\mathrm{AB}}$ are temperature independent but have nonlinear behavior, varying with voltage as

$$
I \propto V^{2 q-1} .
$$

When the temperature exceeds $V$, the response becomes linear. When $V \ll T \ll T_{0}$, both $G_{0}$ and $G_{\mathrm{AB}}$ vary with temperature as 


$$
G \propto\left(\frac{T}{T_{F}}\right)^{2 q-2}
$$

where $T_{\mathrm{F}} \equiv v / a$ is an effective Fermi temperature. This temperature dependence shows that in the strongantidot-coupling regime the renormalization of the electron tunneling amplitudes is not cut off by the finite size $L$ of the antidot edge state, but only by the thermal length $L_{\mathrm{T}}$.

At a temperature near $T_{0}$, we find that $G_{\mathrm{AB}}$ for the CLL displays a pronounced maximum, also in striking contrast to a Fermi liquid [see Fig. 4].

Increasing the temperature further we cross over into the $V \ll T_{0} \ll T$ regime where $G_{0}$ scales as in (84), but

$$
G_{\mathrm{AB}} \propto\left(\frac{T}{T_{0}}\right)\left(\frac{T}{T_{\mathrm{F}}}\right)^{2 q-2} e^{-q T / T_{0}} .
$$

Thus $G_{\mathrm{AB}}$ exhibits a crossover from the well-known $T^{2 q-2}$ Luttinger liquid behavior to a new scaling behavior which is much closer to a chiral Fermi liquid $(q=1)$. Careful measurements in this experimentally accessible regime should be able to distinguish between a Fermi liquid and this predicted nearly Fermi-liquid temperature dependence.

\section{B. High voltage regime: $V \gg T_{0}$}

Again there are three temperature regimes. For the lowest temperatures $T \ll T_{0} \ll V$, the response is again temperature independent and nonlinear. The direct term varies with voltage according to

$$
I_{0} \propto V^{2 q-1}
$$

as in the lowest temperature, low voltage regime. However, the flux-dependent part of the current is now much more interesting, involving power-laws times Bessel functions of the ratio $V / 2 \pi T_{0}=\pi V / \Delta \epsilon$. As an example, for the case $q=3$ we find in this regime

$$
\begin{aligned}
I_{\mathrm{AB}}=\frac{4 e|\Gamma|^{2} a^{4}}{\pi v L^{5}}\{ & {\left[3-\left(\frac{V}{2 \pi T_{0}}\right)^{2}\right] \sin \left(\frac{V}{2 \pi T_{0}}\right) } \\
& \left.-\left[\frac{3 V}{2 \pi T_{0}}\right] \cos \left(\frac{V}{2 \pi T_{0}}\right)\right\},
\end{aligned}
$$

which is shown in Fig. 5 along with the chiral Fermi liquid result (12) at zero temperature. Note that in this low-temperature regime

$$
I_{\mathrm{AB}} \propto\left(\frac{V}{2 \pi T_{0}}\right)^{5 / 2} J_{5 / 2}\left(\frac{V}{2 \pi T_{0}}\right),
$$

where $J$ is a Bessel function of the first kind, a result also obtained by Chamon et al. 25] for the douple pointcontact geometry.
As the temperature is increased further to $T_{0} \ll T \ll$ $V$, we find a crossover to an interesting high-temperature nonlinear regime. Here $I_{0} \propto V^{2 q-1}$ as before, but now

$$
I_{\mathrm{AB}} \propto\left(\frac{T}{T_{0}}\right)^{q} e^{-q T / T_{0}} V^{q-1} \sin \left(\frac{V}{2 \pi T_{0}}\right) .
$$

Therefore, the nonlinear response at fixed temperature can also be used to distinguish between Fermi liquid and Luttinger liquid behavior, even at relatively high temperatures.

When the temperature exceeds $\mathrm{V}$, the response finally becomes linear. When $T_{0} \ll V \ll T, G_{0}$ scales as in 84) whereas $G_{\mathrm{AB}}$ scales as in (85). Thus at high temperatures the low- and high-voltage regimes behave similarily.

\section{PERSISTENT CURRENT IN A CHIRAL LUTTINGER LIQUID}

In the previous section we have been discussing the transport properties of an edge state that occurs at the boundary of a quantum Hall fluid pierced by an antidot potential. In this section we shall discuss a non-Fermiliquid mesoscopic property of the edge current occurring at this same type of antidot boundary or at the boundary of a FQHE droplet confined in a quantum dot.

In a macroscopic edge state, an equilibrium edge current exists even in the absence of an $\mathrm{AB}$ flux or twisted boundary conditions. The magnitude of this current is universal and in the absence of disorder is given by 47]

$$
I_{\text {edge }}=g \frac{e \omega_{\mathrm{c}}}{4 \pi}+\frac{e \tilde{\epsilon}_{\mathrm{qh}}}{2 \pi},
$$

where $\omega_{\mathrm{c}}$ is the cyclotron frequency and $\tilde{\epsilon}_{\mathrm{qh}}$ is the proper quasihole energy (defined at fixed density) of the Laughlin state at filling factor $g=1 / q$.

We now couple the edge state to an $\mathrm{AB}$ flux $\varphi \equiv \Phi / \Phi_{0}$. The grand-canonical partition function of the mesoscopic edge state factorizes into a zero-mode contribution,

$$
Z^{0}=\sum_{n=-\infty}^{\infty} e^{-g \pi^{2}\left(T_{0} / T\right)(n-\varphi)^{2}}
$$

which depends on $\Phi$, and an irrelevant flux-independent contribution from the nonzero-modes. Here $T_{0}$ is again given by Eqn. (11). Note that if $N_{ \pm}$were restricted to be an integer then the period of these equilibrium AB oscillations would be $\Phi_{0} / g$. The allowed fractionally charged excitations (46) are therefore responsible for restoring the AB period to $\Phi_{0}$, as is well-known in other contexts [41].

The edge current induced from the additional flux $\Phi$ is

$$
I \equiv-\frac{\partial \Omega}{\partial \Phi}=\frac{2 \pi T}{\Phi_{0}} \sum_{n=1}^{\infty}(-1)^{n} \frac{\sin (2 \pi n \varphi)}{\sinh \left(n q T / T_{0}\right)},
$$

where $\Omega$ is the grand-canonical potential. At zero temperature, this chiral persistent current has an amplitude (with units now restored) 


$$
\bar{I}=g \frac{e v}{L},
$$

where $L$ is the length of the edge state. Note that $\bar{I}$ is renormalized by the electron-electron interactions in precisely the same way as in a nonchiral Luttinger liquid [31]. At temperatures $T \gg T_{0}$ the amplitude decays as

$$
\bar{I} \approx g \frac{e v}{L} e^{-q T / T_{0}} .
$$

Because these persistent currents are chiral, there is no backscattering from impurities and hence no amplitude reduction from weak disorder. The temperature dependence of the orbital magnetic response of a FQHE edge state may therefore be another ideal system to observe non-Fermi-liquid mesoscopic behavior.

\section{DISCUSSION}

We have studied the tunneling through an edge state formed around an antidot in the fractional quantum Hall effect regime using chiral Luttinger liquid theory. Our analysis has shown that the quantum-point-contact and antidot geometries are considerably different: (i) First, mesoscopic effects are important in the antidot geometry when the thermal length becomes smaller than the size of the antidot, and this leads to a crossover from the powerlaw tunneling characteristics normally associated with a Luttinger liquid to a Fermi-liquid-like scaling. Therefore, mesoscopic effects in a Luttinger liquid can mimic Fermiliquid behavior. This has been demonstrated explicitly for the strong-antidot-coupling case, but it is clear that a similar crossover must occur for all values of the antidot tunneling amplitudes. (ii) The second difference is that because of the unusual nature of the AharonovBohm interference process in a chiral system, there are never perfect resonances in the antidot system, even at zero temperature. This means that at low enough temperatures the system will always be in the strong-antidotcoupling regime, for all values of the Aharonov-Bohm flux and gate voltages. The sharp non-Fermi-liquid resonance studied in the quantum-point-contact geometry, having a width varying with temperature as $T^{1-g}$, is therefore not expected in the antidot geometry at the lowest temperatures.

We have also identified a new experimentally realizable regime, the strong-antidot-coupling regime, where striking non-Fermi-liquid mesoscopic transport phenomena are predicted. This regime is ideal for experimental investigation because the exact current-voltage relation is known [for example, Eqns. (79) through (81)], and the low-temperature crossover from weak-antidot-coupling to strong-antidot-coupling does not complicate the analysis. If, by an appropriate choice of gate voltages, the antidot system starts in the strongly coupled regime, then it will stay in this regime throughout the experimentally relevant ranges of temperature and magnetic field.
Finally, we have predicted new mesoscopic edgecurrent oscillations or "chiral persistent currents" that have a universal non-Fermi-liquid temperature dependence and may be another means to observe a chiral Luttinger liquid.

\section{ACKNOWLEDGMENTS}

This work has been supported by NSERC of Canada. It is a pleasure to thank Claudio Chamon, Chris Ford, John Franklin, Denise Freed, Steve Girvin, Vladimir Goldman, Duncan Haldane, Jung Hoon Han, George Kirczenow, Ilari Maasilta, Kyungsun Moon, David Thouless, and Ulrich Zülicke for useful discussions. M.G. would like to acknowledge the kind hospitality of the Aspen Center for Physics and the University of Washington where some of this work was carried out.

\section{APPENDIX A: QUANTUM MECHANICS OF THE NONINTERACTING CHIRAL ELECTRON GAS}

In this appendix we summarize properties of the chiral Fermi liquid that are needed in the body of the paper. In the presence of a dimensionless $\mathrm{AB}$ flux $\varphi \equiv \Phi / \Phi_{0}$, the single-particle Hamiltonian for right $(+)$ or left $(-)$ movers is

$$
H= \pm v\left(p-\frac{2 \pi \varphi}{L}\right),
$$

with eigenfunctions $\phi_{ \pm}^{n}(x)=L^{-\frac{1}{2}} e^{2 \pi i n x / L}$ and eigenvalues $\pm(n-\varphi) \Delta \epsilon$, where $n$ is an integer, $\Delta \epsilon \equiv 2 \pi v / L$ is the level spacing, and where periodic boundary conditions on a line of length $L$ have been used.

The retarded Green's function

$$
G_{ \pm}^{\mathrm{R}}(x, t) \equiv-i\left\langle\left\{\psi_{ \pm}(x, t), \psi_{ \pm}^{\dagger}(0)\right\}\right\rangle \theta(t)
$$

for a free chiral electron gas has an especially simple form, namely

$$
G_{ \pm}^{\mathrm{R}}(x, t)=-i \theta(t) e^{ \pm 2 \pi i \varphi v t / L} \sum_{n=-\infty}^{\infty} \delta(x \mp v t+n L) .
$$

An electron added to the system therefore propagates ballistically with a velocity $v$ and with no dispersion. The Fourier transform of $G_{ \pm}^{\mathrm{R}}(x, t)$ is

$$
\begin{aligned}
G_{ \pm}^{\mathrm{R}}(x, \omega) & =\frac{1}{L} \sum_{n=-\infty}^{\infty} \frac{e^{2 \pi i n x / L}}{\omega \mp(n-\varphi) \Delta \epsilon+i \eta} \\
& =-\frac{i}{v} \sum_{n=-\infty}^{\infty} \theta( \pm(x+n L)) e^{2 \pi i\left(\frac{\omega}{\Delta \epsilon} \pm \varphi\right)\left(\frac{x}{L}+n\right)} .
\end{aligned}
$$


The second form (A5) follows from (A4) by an application of the Poisson summation formula, or from (A3) directly. The second expression has a useful interpretation: $G_{ \pm}^{\mathrm{R}}(x, \omega)$ is proportional to the amplitude for an electron to propagate a distance $x$ around the ring via a direct path, during which it acquires a phase

$$
\exp \left[i\left(\frac{2 \pi \omega}{\Delta \epsilon} \pm 2 \pi \varphi\right) \frac{x}{L}\right]
$$

plus the amplitude to propagate via any number of windings around the ring with a given chirality. The first term in (A6) is the dynamical phase, whereas the second term is the chirality-dependent AB phase.

The total amplitude to propagate at frequency $\omega$ from point 1 to point 2 on the ring shown in Fig. 1, allowing only clockwise $(+)$ or counterclockwise $(-)$ motion, is proportional to

$$
G_{ \pm}^{\mathrm{R}}\left(\frac{L}{2}, \omega\right)=\frac{1}{2 v \sin \pi\left(\frac{\omega}{\Delta \epsilon} \pm \varphi\right)} .
$$

Note that the chirality enters only through the AB phase. The transmission probability is proportional to

$$
\begin{aligned}
& \left|G_{ \pm}^{\mathrm{R}}\left(\frac{L}{2}, \omega\right)\right|^{2}=\frac{1 / 2 v^{2}}{1-\cos 2 \pi\left(\frac{\omega}{\Delta \epsilon} \pm \varphi\right)} \\
& G_{ \pm}^{(m)}(x, \tau)=\left\langle e^{i m\left(\phi_{ \pm}^{0}(x, \tau)-\phi_{ \pm}^{0}(0)\right)}\right\rangle\left(e^{\frac{m^{2}}{2}\left[\phi_{ \pm}^{0}(x, \tau), \phi_{ \pm}^{0}(0)\right]} e^{m^{2} g \mathcal{S}( \pm x+i v \tau)} \theta(\tau)+e^{i \pi m^{2} g} e^{-\frac{m^{2}}{2}\left[\phi_{ \pm}^{0}(x, \tau), \phi_{ \pm}^{0}(0)\right]} e^{m^{2} g \mathcal{S}(\mp x-i v \tau)} \theta(-\tau)\right)
\end{aligned}
$$

which possesses transmission resonances when $\frac{\omega}{\Delta \epsilon} \pm \varphi$ is integral, but never exhibits completely destructive interference.

For completeness we also give expressions for the spectral function $A_{ \pm}(k, \omega) \equiv-2 \operatorname{Im} G_{ \pm}^{\mathrm{R}}(k, \omega)$ and density of states $N_{ \pm}(\omega) \equiv \int_{-\infty}^{\infty} \frac{d k}{2 \pi} A_{ \pm}(k, \omega)$ for free chiral fermions in the infinite system-size limit: $A_{ \pm}(k, \omega)=2 \pi \delta(\omega \mp v k)$ and $N_{ \pm}(\omega)=1 / v$.

\section{APPENDIX B: EUCLIDIAN QUASIPARTICLE PROPAGATOR}

Here we use the results of Section III to calculate the $m$-quasiparticle propagator for a finite-size CLL with $\varphi=0$, as defined in Section IV. This propagator may be written in terms of the quantized chiral scalar field (23) as

$$
G_{ \pm}^{(m)}(x, \tau) \equiv\left\langle T e^{i m \phi_{ \pm}(x, \tau)} e^{-i m \phi_{ \pm}(0)}\right\rangle
$$

where $T$ is the time-ordering operator for particles with fractional statistics $e^{i \pi m^{2} g}$. The imaginary-time equa-

where $e^{i \pi m^{2} g}$ is the statistical phase of the $m$-quasiparticle composite and

$$
\mathcal{S}(x) \equiv \frac{2 \pi}{L} \sum_{k>0} \frac{e^{i k x}-1}{k} e^{-k a} .
$$

The quantity $\mathcal{S}(x)$ may be found by differentiating with respect to $x$, performing the summation, and then integrating, which yields

$$
\mathcal{S}(x)=\ln \left(\frac{i \pi a / L}{\sin [\pi(x+i a) / L]}\right)-\frac{i \pi x}{L} .
$$

The second term in (4) leads to a cancellation of the zero-mode commutators in (2). The remaining zero-mode expectation value, which has the form

$$
\left\langle e^{ \pm 2 \pi i m N_{ \pm}(x \pm i v \tau) / L}\right\rangle=\frac{\sum_{n} e^{-\beta \pi v g n^{2} / L} e^{ \pm 2 \pi i m n g(x \pm i v \tau) / L}}{\sum_{n} e^{-\beta \pi v g n^{2} / L}}
$$

is equal to unity in the zero-temperature limit, so the final result is

$$
G_{ \pm}^{(m)}(x, \tau)=\left(\frac{ \pm i \pi a / L}{\sin [\pi(x \pm i v \tau \pm i a \operatorname{sgn} \tau) / L]}\right)^{m^{2} g}
$$

where the branch cut has been chosen to cancel the statistical phase. 
[1] The Quantum Hall Effect, edited by R. E. Prange and S. M. Girvin (Springer-Verlag, New York, 1990).

[2] For a review see C. W. J. Beenakker and H. van Houten in Solid State Physics: Advances in Research and Applications, edited by H. Ehrenreich and D. Turnbull (Academic Press, New York, 1991), Vol. 44.

[3] B. I. Halperin, Phys. Rev. B 25, 2185 (1982).

[4] M. Büttiker, Phys. Rev. Lett. 57, 1761 (1986); P. Streda, J. Kucera, and A. H. MacDonald, Phys. Rev. Lett. 59, 1973 (1987); J. K. Jain and S. A. Kivelson, Phys. Rev. Lett. 60, 1542 (1988); M. Büttiker, Phys. Rev. B 38, 9375 (1988).

[5] R. B. Laughlin, Phys. Rev. Lett. 50, 1395 (1983).

[6] X. G. Wen, Phys. Rev. Lett. 64, 2206 (1990); Phys. Rev. B 41, 12838 (1990); Phys. Rev. B 43, 11025 (1991).

[7] S. M. Girvin, in Chap. 10 of Ref. 11; S. M. Girvin and A. H. MacDonald, Phys. Rev. Lett. 58, 1252 (1987); S. C. Zhang, T. H. Hansson, and S. A. Kivelson, Phys. Rev. Lett. 62, 82 (1989); J. Fröhlich and A. Zee, Nuc. Phys. B 364, 517 (1991); A. Lopez and E. Fradkin, Phys. Rev. B 44, 5246 (1991); B. I. Halperin, P. A. Lee, and N. Read, Phys. Rev. B 47, 7312 (1993).

[8] For reviews, see J. Sólyom, Adv. Phys. 28, 201 (1979), and V. J. Emery, in Highly Conducting One-Dimensional Solids, edited by J. T. Devreese, R. P. Evrard, and V. E. van Doren (Plenum, New York, 1979).

[9] F. D. M. Haldane, J. Phys. C 14, 2585 (1981).

[10] For reviews, see X. G. Wen, Int. J. Mod. Phys. B 6, 1711 (1992), and X. G. Wen, Adv. Phys. 44, 405 (1995).

[11] C. L. Kane and M. P. A. Fisher, Phys. Rev. Lett. 68, 1220 (1992); Phys. Rev. B 46, 15233 (1992).

[12] C. de C. Chamon and X. G. Wen, Phys. Rev. Lett. 70, 2605 (1993).

[13] K. Moon, H. Yi, C. L. Kane, S. M. Girvin, and M. P. A. Fisher, Phys. Rev. Lett. 71, 4381 (1993).

[14] V. L. Pokrovsky and L. P. Pryadko, Phys. Rev. Lett. 72, 124 (1994).

[15] C. L. Kane, M. P. A. Fisher, and J. Polchinski, Phys. Rev. Lett. 72, 4129 (1994).

[16] C. de C. Chamon, D. E. Freed, and X. G. Wen, Phys. Rev. B 51, 2363 (1995); Phys. Rev. B 53, 4033 (1996).

[17] P. Fendley, A. W. W. Ludwig, and H. Saleur, Phys. Rev. Lett. 74, 3005 (1995); Phys. Rev. B 52, 8934 (1995).

[18] Y. Oreg and A. M. Finkel'stein, Phys. Rev. Lett. 74, 3668 (1995).

[19] C. L. Kane and M. P. A. Fisher, Phys. Rev. B 51, 13449 (1995); Phys. Rev. B 51, 13449 (1995); Phys. Rev. B 52, 17393 (1995).

[20] S. R. Renn and D. P. Arovas, Phys. Rev. B 51, 16832 (1995).

[21] A. V. Balatsky and S. I. Matveenko, Phys. Rev. B 52, 8676 (1995).

[22] J. J. Palacios and A. H. MacDonald, Phys. Rev. Lett. 76, 118 (1996).

[23] K. Moon and S. M. Girvin, Phys. Rev. B 54, 4448 (1996).
[24] M. R. Geller, D. Loss, and G. Kirczenow, Phys. Rev. Lett. 77, 5110 (1996).

[25] C. de C. Chamon, D. E. Freed, S. A. Kivelson, S. L. Sondhi, and X. G. Wen, Phys. Rev. B 55, 2331 (1997).

[26] F. P. Milliken, C. P. Umbach, and R. A. Webb, Solid State Commun. 97, 309 (1996).

[27] A. M. Chang, L. N. Pfeiffer, and K. W. West, Phys. Rev. Lett. 77, 2538 (1996).

[28] J. D. F. Franklin et al., Surf. Sci. 361, 17 (1996).

[29] I. J. Maasilta and V. J. Goldman, Phys. Rev. B 55, 4081 (1997).

[30] V. J. Goldman and B. Su, Science 267, 1010 (1995).

[31] D. Loss, Phys. Rev. Lett. 69, 343 (1992); D. Loss and D. Maslov, in "Quantum Dynamics of Submicron Structures", edited by H. A. Cerdeira, B. Kramer and G. Schön, NATO ASI Series E, Vol. 291 (Kulwer, Dordrecht, 1995), 199-210.

[32] F. V. Kusmartsev, JETP Lett. 60, 649 (1994); R. Fazio, F. W. J. Hekking, and A. A. Odintsov, Phys. Rev. Lett. 74, 1843 (1995); G. Gómes-Santos, Phys. Rev. Lett. 76, 4223 (1996); S. R. Renn and D. P. Arovas (preprint).

[33] Y. Aharonov and D. Bohm, Phys. Rev. 115, 485 (1959).

[34] A. G. Aronov and Yu. V. Sharvin, Rev. Mod. Phys. 59, 755 (1987).

[35] G. Kirczenow, Phys. Rev. B 50, 1649 (1994).

[36] D. R. Mace et al., Phys. Rev. B 52, 8672 (1995).

[37] W. C. Tan and J. C. Inkson, Phys. Rev. B. 53, 6947 (1996).

[38] P. J. Simpson et al., Appl. Phys. Lett. 63, 3191 (1993); P. J. Simpson et al., Surf. Sci. 305, 453 (1994); C. J. B. Ford et al., Phys. Rev. B 49, 17456 (1994).

[39] The antidot tunneling amplitudes $\Gamma_{ \pm}=i \Gamma$ are chosen to be purely imaginary so that in the $\Gamma \rightarrow 0$ limit the electron suffers no phase shift at the tunneling points.

[40] G. Kirczenow, Phys. Rev. B 53, 15767 (1996). See also G. Kirczenow and B. L. Johnson, Phys. Rev. B 51, 17579 (1995).

[41] P. A. Lee, Phys. Rev. Lett. 65, 2206 (1990); S. A. Kivelson, Phys. Rev. Lett. 65, 3369 (1990); D. J. Thouless and Y. Gefen, Phys. Rev. Lett. 66, 806 (1991).

[42] K. A. Matveev, D. Yue, and L. I. Glazman, Phys. Rev. Lett. 71, 3351 (1993).

[43] We define resonance here in the sense it is used in the Luttinger liquid literature: The system is on resonance when the AB flux inhibits transmission through the antidot. Thus, CLL theory at low temperature predicts peaks in the two-terminal conductance on resonance, and vanishing conductance elsewhere. This definition is opposite to what is usually used in the antidot and $\mathrm{AB}$ effect literature, which defines resonance to occur when the flux allows tunneling through the antidot.

[44] We shall not attempt a derivation of (52) here, which is complicated by the fact that the nonlocal commutation relation (21) invalidates the usual construction of a basis of field eigenstates as products of simultaneous eigenstates at each point.

[45] In the strong-antidot-coupling regime, however, the renormalization of the tunneling amplitudes is not cut off by $L$, but only by the finite temperature (or finite voltage). This is because in this regime the geometry is 
effectively that of two infinite edge states as shown in Fig. 2b.

[46] X. G. Wen, Phys. Rev. B 44, 5708 (1991).
[47] M. R. Geller and G. Vignale, Phys. Rev. B 52, 14137 (1995).

FIG. 1. Mesoscopic ring. $\theta_{ \pm}$is the phase shift subjected to an electron of energy $\epsilon$ after a complete orbit in the clockwise $(+)$ or counterclockwise $(-)$ direction.

FIG. 2. Aharonov-Bohm effect geometry in the (a) weak-antidot-coupling and (b) strong-antidot-coupling regimes. In both cases the arrows denote the direction of currents and the dashed lines represent weak tunneling processes.

FIG. 3. Zero-temperature Aharonov-Bohm resonances in the chiral Fermi liquid with weak coupling $(\Gamma=0.2$, solid curve), intermediate coupling $(\Gamma=0.5$, dashed curve), and with strong coupling $(\Gamma=0.9$, dotted curve). The source-drain conductance $G$ is given in units of $e^{2} / h$ and the flux is in units of the flux quantum $\Phi_{0}$.

FIG. 4. Temperature dependence of $G_{\mathrm{AB}}$ for the cases $g=1$ (dashed curve) and $g=1 / 3$ (solid curve). Both curves are normalized to have unit amplitude at their respective maxima.

FIG. 5. Nonlinear IV curve for the the cases $g=1$ (dashed curve) and $g=1 / 3$ (solid curve). The current is in arbitrary units and $V_{0} \equiv \Delta \epsilon / \pi$. 